\title{
Protein Degradation Process
}

National Cancer Institute

\section{Source}

National Cancer Institute. Protein Degradation Process. NCI Thesaurus. Code C18104.

Specific degradation of a protein - involving conjugation, transport, oxidation, and

proteolysis - involved in signal transduction or cell communication events; not to be

confuse with proteolysis, which applies only to peptide bond hydrolysis. $(\mathrm{NCl})$ 